\title{
Minimum Redundancy MIMO Array Synthesis by means of Cyclic Difference Sets
}

\author{
Jian Dong, Ronghua Shi, Wentai Lei, and Ying Guo \\ School of Information Science and Engineering, Central South University, Changsha 410083, China \\ Correspondence should be addressed to Jian Dong; dongjian@mail.csu.edu.cn
}

Received 18 February 2013; Accepted 14 August 2013

Academic Editor: Wenhua Chen

Copyright (C) 2013 Jian Dong et al. This is an open access article distributed under the Creative Commons Attribution License, which permits unrestricted use, distribution, and reproduction in any medium, provided the original work is properly cited.

\begin{abstract}
Antenna array synthesis is an important issue in MIMO radars. By judiciously designing antenna positions, one can create a very long virtual array steering vector with a small number of antennas and therefore achieve very high spatial resolution at a small cost. This paper presents a combinatorial methodology based on cyclic difference sets (CDSs) for minimum redundancy (MR) MIMO array synthesis which seeks to maximize the virtual array aperture for a given number of antennas. First, the key features of CDSs and the CDS-based MR-MIMO layouts are described. Then, the analytical expression of the maximum contiguous virtual array aperture is derived. Further, based on this expression, an enumerative shifting procedure is developed for identifying the optimal CDS-based MR-MIMO layout. Selected examples are analyzed to point out the computational effectiveness of the CDS-based MRMIMO array synthesis.
\end{abstract}

\section{Introduction}

Multiple-input multiple-output (MIMO) radars have drawn remarkable attention recently for high spatial resolution, reducing vulnerability and overcoming fading effect [1-7]. MIMO radars transmit orthogonal waveforms or noncoherent waveforms instead of transmitting coherent waveforms in traditional transmitter based radars. In the MIMO radar receiver, a matched filter bank is used to extract the orthogonal waveform components. There are two different kinds of MIMO radars using the noncoherent waveforms. The first is the statistical MIMO radars for increased spatial diversity $[2,3]$. In this scenario, all antennas are far enough from each other so that they obtain echoes from different angles of target to combat target fades. The second is the colocated MIMO radars for a better spatial resolution [4-7]. In this scenario, the distances between transmitting antennas are small enough compared to the distance between the target and the radar station. Therefore, the target RCS is identical for all transmitting paths. The phase differences caused by different transmitting antennas along with the phase differences caused by different receiving antennas can form a new virtual array steering vector. With judiciously designed antenna positions, one can create a very long array steering vector with a small number of antennas. Thus, the spatial resolution can be dramatically increased at a small cost. In this paper, we focus on this second advantage.

In fact, the problem of how to properly arrange the transmitting and receiving $(\mathrm{Tx} / \mathrm{Rx})$ antenna elements in order to achieve the optimal spatial resolution has received considerable interests. As we all know, the uniform $\mathrm{Tx} / \mathrm{Rx}$ arrays will lead to a uniform virtual array with a large amount of redundant spacings. Several thinned $\mathrm{Tx} / \mathrm{Rx}$ array configurations were given by Forsythe and Bliss $[1,4]$ and Wang $[7,8]$ to reduce redundant spacings in the virtual array. A similar MIMO array design problem was considered in medical ultrasound imaging and direction of arrival estimation from the viewpoint of coarray $[9,10]$, and also several classes of thinned MIMO array configurations were presented. In [11], Chen and Vaidyanathan extended the minimum redundancy (MR) idea [12, 13] originally used in the field of radio astronomy and spectrum estimation to MIMO radars and formulated the MIMO array design as a problem of MRMIMO array synthesis, which is to properly place $\mathrm{Tx} / \mathrm{Rx}$ elements in order to make the contiguous aperture of the virtual array as large as possible. 
The search of MR-MIMO arrays is difficult because the inverse mapping from difference coarray of the virtual array to $\mathrm{Tx} / \mathrm{Rx}$ array configurations is analytically unknown. For a very small number of Tx/Rx elements, an exhaustive search [11] or a stochastic optimizer (e.g., genetic algorithms (GAs) and particle swarm optimization (PSO) [14]) is usually used. However, for a modest or large number of $\mathrm{Tx} / \mathrm{Rx}$ elements, these algorithms do not seem feasible due to the exponentially explosive search space. Recently, some classes of analytical binary sequences, such as cyclic difference sets (CDSs) [15, 16] and almost difference sets (ADSs) [17-22], have been applied to antenna array pattern synthesis. The analytical methodologies present several potentials over other stateof-the-art approaches since (1) they are not affected by convergence problems regardless of the array size; (2) the array performances (e.g., peak sidelobe levels) are theoretically controlled or predicted for any aperture; (3) these binary sequences may be an effective way to enhance global optimization methods. For MIMO applications, CDSs have been firstly exploited by the authors [23] to synthesize MRMIMO arrays. In [23], we demonstrated that by placing the $\mathrm{Tx} / \mathrm{Rx}$ elements at the CDS-derived locations, a virtual array with a large contiguous aperture can be synthesized using a very small number of Tx/Rx elements. However, considering the large amount of equivalent CDSs for the cyclic shift property, there exist a large number of different CDS-based MR-MIMO layouts with different virtual array apertures. So, a suitable procedure is needed for identifying the optimal CDS-based MR-MIMO layout. In this paper, following the developments of [23], we derive the analytical expression of the maximum contiguous virtual array aperture of CDSbased MR-MIMO layouts. Then, based on this, we develop an enumerative shifting procedure for identifying the optimal CDS-based MR-MIMO layout. This work can be considered as the complement and extension of the CDS-based MRMIMO framework established in [23].

The rest of the paper is organized as follows. In Section 2, the problem of MR-MIMO array synthesis is mathematically formulated from the viewpoint of coarray. In Section 3, based on the key features of CDSs, the CDS-based MR-MIMO arrays are firstly presented. Then, based on the analytical expression of the maximum contiguous virtual array aperture, an enumerative shifting procedure is carefully described for identifying the optimal CDS-based MR-MIMO layout. In Section 4, representative numerical results are provided to illustrate the effectiveness of the MR-MIMO array synthesis by means of CDSs. Finally, Section 5 sums up the paper and presents conclusions.

\section{Problem Formulation of MR-MIMO Array Synthesis}

Consider a colocated MIMO radar with an $M$-element linear transmitting antenna array and an $N$-element linear receiving antenna array. Let $x_{T i}$ and $x_{R j}$ denote the position of the $i$ th transmitting and the $j$ th receiving antennas normalized by unit spacing (usually half wavelength), respectively, where $i=0,1, \ldots, M-1$ and $j=1,2, \ldots, N-1$. By transmitting orthogonal waveforms and extracting the waveforms in each receiving element with a set of matched filters, a virtual receiving array with $N M$ antenna elements located at $[1,4]$

$$
V A=\left\{x_{T i}+x_{R j} \mid i=0,1, \ldots, M-1 ; j=0,1, \ldots, N-1\right\}
$$

can be synthesized. Correspondingly, the difference coarray (i.e., the spacing set) of the virtual array can be expressed as

$$
\begin{array}{r}
D_{V A}=\left\{x_{T i}+x_{R j}-\left(x_{T i^{\prime}}+x_{R j^{\prime}}\right) \mid i, i^{\prime}=0,1, \ldots, M-1 ;\right. \\
\left.j, j^{\prime}=0,1, \ldots, N-1\right\} .
\end{array}
$$

In MR-MIMO array design, the idea is to form a coarray which is minimally redundant in spacings, that is, a coarray that captures all of the spacings with a minimum number of $\mathrm{Tx} / \mathrm{Rx}$ element pairs (ideally, each element of a nonredundant coarray involves only a single $\mathrm{Tx} / \mathrm{Rx}$ element pair). The completeness of the coarray is required in order that a good point spread function (PSF) for imaging applications can be obtained $[4,9]$ or a covariance matrix can be estimated for direction of arrival estimation or adaptive beamforming $[10,13]$.

For a given total number of $\mathrm{Tx} / \mathrm{Rx}$ elements, the MRMIMO array design can be generalized as a multivariable constraint optimization problem [23] as follows:

$$
\begin{aligned}
& \max _{\left\{x_{T i}\right\},\left\{x_{R j}\right\}} L \\
& \text { s.t. } H\left(D_{V A}\right)=0, \quad\left|\left\{x_{T i}\right\}\right|=M, \quad\left|\left\{x_{R j}\right\}\right|=N \text {, }
\end{aligned}
$$

where the objective function $L$ is the largest contiguous aperture of the virtual array in MIMO radars; $|A|$ denotes the cardinality of the set $A$; the constraint function $H\left(D_{V A}\right)$ denotes the number of holes (i.e., missing spacings) in the coarray and can be computed by

$$
H\left(D_{V A}\right)=\sum_{s=1}^{L}\left(s \in D_{V A}\right),
$$

where $\left(s \in D_{V A}\right)$ is equal to 0 if $s$ belongs to $D_{V A}$ and 1 otherwise.

The difficulty in solving this problem is that the inverse mapping from the difference coarray of the virtual array to $\mathrm{Tx} / \mathrm{Rx}$ array configurations is analytically unknown. Usually, a numerical implementation described as follows is preferable. Given transmitting element number $M$ and receiving element number $N$, an initial maximum aperture $L$ of the synthesized virtual array is chosen to determine whether the $\mathrm{Tx} / \mathrm{Rx}$ elements can be placed at the proper locations so that all the required spacings of the coarray are present; that is, $H\left(D_{V A}\right)=0$. If such $\mathrm{Tx} / \mathrm{Rx}$ configurations are found, $L$ can be increased, and the procedure is repeated until $L$ grows too large. For such a numerical implementation, stochastic optimization techniques (e.g., PSO in [14]) seem good candidates because of their flexibility and generality. Usually, they allow 
to effectively explore the whole solution space and to figure out optimal MIMO array layout. However, it is very difficult to find a satisfactory solution after a nonnegligible number of iterations due to the exponentially explosive solution space, especially for modest or large MIMO array designs. Fortunately, the introduction of analytical binary sequences known as cyclic difference sets (CDSs) [23] presents a deterministic thinning methodology for MR-MIMO array synthesis. This CDS-based methodology can determine the analytical locations of $\mathrm{Tx} / \mathrm{Rx}$ array elements and therefore is not affected by convergence problems regardless the array size. In this paper, starting from these CDS-derived MIMO layouts, the maximum contiguous virtual array aperture $L$ will be theoretically derived for any given number of $\mathrm{Tx} / \mathrm{Rx}$ antennas. With this analytical expression of $L$, the optimization problem in (3) can be greatly simplified. Then, the identifying of the optimal MR-MIMO layout will be limited to an enumerative shifting of reference sequences available in Web repositories [24].

\section{Minimum Redundancy MIMO Array Synthesis by means of Cyclic Difference Sets}

3.1. CDS-Based MR-MIMO Array. CDSs are combinatorial methods initially investigated in combinatorial mathematics and information theory $[25,26]$ and later applied to antenna array optimization $[15,16,23]$. A CDS $D(V, K, \Lambda)$ is a set of $K$ integers $\left\{d_{i}\right\}[25,26]$

$$
D=\left\{d_{0}, d_{1}, \ldots, d_{K-1}\right\}, \quad \text { with } 0 \leq d_{i}<V
$$

such that any integer $v(0<v<V)$ can be represented exactly $\Lambda$ times in the form $v \equiv d_{j}-d_{i}(\bmod V)$, where " $\bmod V$ " means the difference is to be taken modulo $V$. The parameters $(V, K, \Lambda)$ of any CDS are connected by

$$
K(K-1)=\Lambda(V-1),
$$

and so there are only two independent parameters among $V$, $K$, and $\Lambda$. Given a $\operatorname{CDS} D(V, K, \Lambda)$, the set

$$
D^{\prime}=\left\{d_{0}+s, d_{1}+s, \ldots, d_{K-1}+s\right\} \equiv D+s,
$$

where each element is taken modulo $V$, will also be a $(V, K, \Lambda)$ difference set. In this case, $D^{\prime}$ is called a cyclic shift of $D$. If $D_{p}$ and $D_{q}$ are two difference sets with the same parameters $(V, K, \Lambda)$ and $D_{p}=t D_{q}+s$ for any integers $t$ and $s$ with $t$ prime to $V$, then $D_{p}$ and $D_{q}$ are called equivalent difference sets.

In [23], we demonstrated that by placing the $\mathrm{Tx} / \mathrm{Rx}$ elements at the CDS-derived locations, a very large virtual array can be synthesized with a small number of $\mathrm{Tx} / \mathrm{Rx}$ elements. The $M \times N$ CDS-based MR-MIMO arrays are constructed as follows:

$$
\begin{gathered}
\left\{x_{T m}\right\}=\left\{d_{m}\right\}, \\
\left\{x_{R n}\right\}=\left\{b_{n} \cdot V\right\},
\end{gathered}
$$

where $\left\{d_{m}\right\}$ is a CDS with the parameters $(V, M, 1), m=$ $0,1, \ldots, M-1 ; d_{0}=0$ is specified; $\Lambda=1$ is specified to ensure that the synthesized contiguous aperture $L$ is as large as possible; $\left\{b_{n}\right\}$ is a difference basis for a $[0, P]$ segment, $n=0,1, \ldots, N-1 ; b_{0}=0$ is specified. A difference basis for the $[0, P]$ segment is a set of $N$ integers $\left\{b_{i}\right\}[27,28]$ :

$$
B=\left\{b_{0}, b_{1}, \ldots, b_{N-1}\right\}, \quad \text { with } 0 \leq b_{i} \leq P
$$

such that any integer from this segment can be expressed as a difference $b_{j}-b_{i}$. In fact, an $N$-element difference basis corresponds to an $N$-element minimum redundancy linear array in the transmitting only or receiving only scenario.

3.2. Analytical Expression of the Maximum Contiguous Aperture of Virtual Array. From (1), (8), and (9), the synthesized virtual array determined by the CDS-based approach can be expressed as

$$
V A=\left\{d_{m}+b_{n} \cdot V\right\} .
$$

Note that the uniformity of its difference coarray (i.e., the constraint $\left.H\left(D_{V A}\right)=0\right)$ is naturally guaranteed by the definitions of both CDSs and difference bases [23]. Now, we will derive the maximum contiguous aperture of $V A$, that is, looking for a maximum value of $L$ such that every integer $\xi$ $(0 \leq \xi \leq L)$ can be expressed as the difference between two integers $d_{m}+b_{n} \cdot V$ and $d_{m^{\prime}}+b_{n^{\prime}} \cdot V$ of the set $V A$.

To show this, we put $\xi=\xi_{1} \cdot V+\xi_{2}$, where $0 \leq \xi_{1} \leq P-1$ and $0 \leq \xi_{2} \leq V-1$. If $\xi_{2}$ is expressible as $\xi_{2}=d_{m}-d_{m^{\prime}}$, we choose $b_{n}, b_{n^{\prime}}$ such that $\xi_{1}=b_{n}-b_{n^{\prime}}$; then,

$$
\begin{aligned}
\xi & =\left(b_{n}-b_{n^{\prime}}\right) \cdot V+d_{m}-d_{m^{\prime}} \\
& =\left(d_{m}+b_{n} \cdot V\right)-\left(d_{m^{\prime}}+b_{n^{\prime}} \cdot V\right) .
\end{aligned}
$$

If, however, $\xi_{2}$ is expressible as $\xi_{2}-V=d_{m}-d_{m^{\prime}}$, we choose $b_{n}, b_{n^{\prime}}$ such that $\xi_{1}+1=b_{n}-b_{n^{\prime}}$; then

$$
\begin{aligned}
\xi & =\left(b_{n}-b_{n^{\prime}}-1\right) \cdot V+d_{m}-d_{m^{\prime}}+V \\
& =\left(d_{m}+b_{n} \cdot V\right)-\left(d_{m^{\prime}}+b_{n^{\prime}} \cdot V\right) .
\end{aligned}
$$

Also if $b_{n}-b_{n^{\prime}}=P$,

$$
P V=\left(d_{m}+b_{n} \cdot V\right)-\left(d_{m}+b_{n^{\prime}} \cdot V\right) .
$$

Thus the maximum contiguous aperture $L$ can reach $P V$, and the set $V A$ is a difference basis with respect to $P V$.

In the set (8) we observe that no integer $\xi$ such that $d_{\max }-d_{\min }<\xi<V$ is expressible in the form $d_{m}-d_{m^{\prime}}$, where $d_{\max }$ and $d_{\min }$ are the largest element and the smallest element, respectively. Consequently the integers

$$
1,2, \ldots, V-\left(d_{\max }-d_{\min }\right)-1
$$

are expressible in the form $d_{m}-d_{m^{\prime}}$. Choosing $b_{n}, b_{n^{\prime}}$ such that $b_{n}-b_{n^{\prime}}=P$, we express the integers

$$
P V+1, P V+2, \ldots, P V+V-\left(d_{\max }-d_{\min }\right)-1
$$

as differences $\left(d_{m}+b_{n} \cdot V\right)-\left(d_{m^{\prime}}+b_{n^{\prime}} \cdot V\right)$ between pairs of integers of the set $V A$. Therefore, the maximum contiguous aperture of the virtual array is determined by

$$
L=(P+1) V-\left(d_{\max }-d_{\min }\right)-1 .
$$


3.3. Optimal MR-MIMO Array by Enumerative Shifting Procedure. Considering the large amount of equivalent CDSs for the cyclic shift property, there are always a large number of different CDS-based MIMO layouts $\left\{d_{m}+b_{n} \cdot V\right\}$ with different virtual array apertures $L$ even for a given number of $\mathrm{Tx} / \mathrm{Rx}$ antennas. Expression (17) indicates that the optimal MR-MIMO layout with the maximum $L$ will be achieved by selecting the CDS with the minimum value of $d_{\max }-d_{\min }$. Towards this end, the problem of identifying the optimal MRMIMO layout among all existing CDS-based layouts for a given number of $\mathrm{Tx} / \mathrm{Rx}$ antennas is recast as an optimization one where the fitness function $\Phi$ to be minimized is defined as follows:

$$
\Phi(D) \stackrel{\Delta}{=} \operatorname{diff}(D)
$$

where

$$
\operatorname{diff}(D)=d_{\max }-d_{\min }
$$

with $d_{\max }$ and $d_{\min }$ being the largest element and the smallest element of a cyclic difference set $D$, respectively. Obviously, by deriving the analytical expression of the maximum contiguous virtual array aperture $L$, the solving of MR-MIMO array problem in (3) has been simplified as identifying the optimal CDS with minimum fitness $\Phi(D)$.

Inspired by the discrete nature of the descriptors of the CDSs, an enumerative shifting procedure for searching optimal MR-MIMO layout is developed as follows.

(i) CDS selection and initialization: given transmitting antenna number $M$, select a $(V, M, 1)$-CDS from the CDS Web repositories [24] as the initial CDS (where $\Lambda=1$ is specified) as follows:

$$
D^{(0)}=\left\{d_{m}^{(0)} \in Z^{V}, m=0, \ldots, M-1\right\} .
$$

Set $\operatorname{diff}{ }^{\text {opt }}=\operatorname{diff}\left(D^{(0)}\right)=d_{\max }^{(0)}$ (usually, $d_{\min }^{(0)}=0$ is specified in the initial CDS).

(ii) CDS cyclic shift: by exploiting the cyclic shift property, enumerate the $\sigma$-shifted version of the initial CDS and let

$$
D^{(\sigma)}=\left\{d_{m}^{(\sigma)} \in Z^{V}, m=0, \ldots, M-1: d_{m}^{(\sigma)}=\left.\left(d_{m}+\sigma\right)\right|_{\bmod V}\right\},
$$

where $\left.(\cdot)\right|_{\bmod V}$ means modulo operation. Clearly, all these sets are the equivalent sets of the initial CDS.

(iii) Update the fitness: evaluate $\operatorname{diff}\left(D^{(\sigma)}\right)=d_{\max }^{(\sigma)}-d_{\min }^{(\sigma)}$, where $d_{\max }^{(\sigma)}$ and $d_{\min }^{(\sigma)}$ are the largest element and the smallest element in the $\sigma$-shifted CDS $D^{(\sigma)}$, respectively. If $\operatorname{diff}\left(D^{(\sigma)}\right)<$ diff $^{\text {opt }}$, then update $\sigma^{\text {opt }}=\sigma, D^{\text {opt }}=D^{(\sigma)}$, and diff ${ }^{\text {opt }}=$ $\operatorname{diff}\left(D^{(\sigma)}\right)$.

(iv) Convergence check: if $\sigma=V-1$, then return $\sigma^{\mathrm{opt}}$ and terminate; else continue.

(v) Update: update the shift indexes $\sigma$ (i.e., $\sigma \leftarrow(\sigma+$ 1) $\left.\right|_{\bmod V}$ ) to generate a new CDS sequence (i.e., a new cyclic shifted version of the reference CDS chosen in (i)) and go to (ii).

It is worth to notice that such a numerical procedure is very simple and computationally efficient since just up to $V \times$ $M=M^{2} \times(M-1)$ evaluations are required for an $M \times N$ MIMO array.

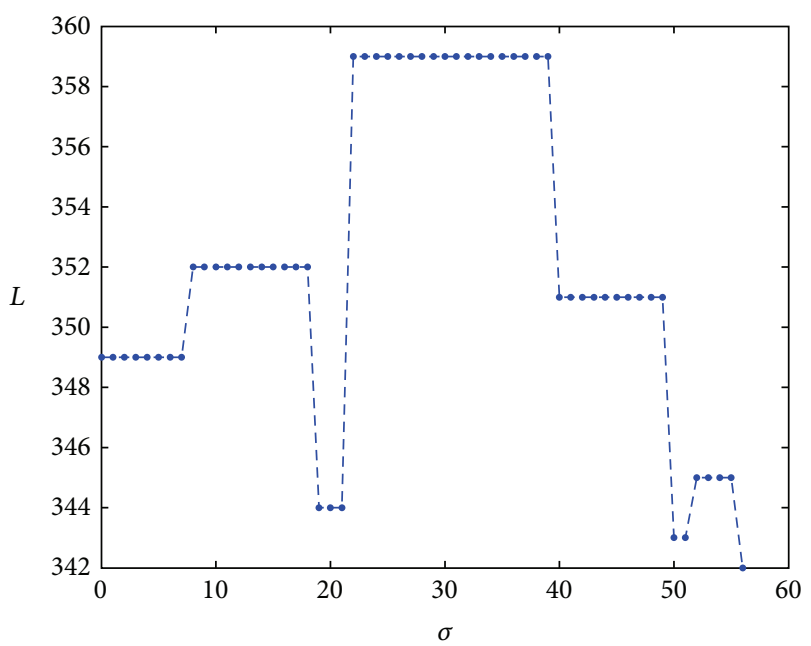

FIGURE 1: CDS-based enumerative shifting procedure: behavior of virtual array aperture $L$ versus evaluated shift $\sigma$ for CDS-based MRMIMO array $(M=8, N=4)$.

\section{Numerical Analysis}

The potentialities of the CDS-based MR-MIMO array synthesis are assessed through a set of numerical experiments aimed at completing and extending the validations presented in [23]. Since the CDS-based planar MR-MIMO array can be easily generated by "multiplying" two orthogonal CDS-based linear MR-MIMO arrays [23], the following numerical studies will be focused on the linear MR-MIMO array synthesis.

The first test case refers to a half wavelength $(\Delta u=0.5 \lambda)$ MIMO radar array with an eight-element linear transmitting array $(M=8)$ and a four-element linear receiving array $(N=$ 4 ). The synthesis of an $8 \times 4 \mathrm{MR}-\mathrm{MIMO}$ array is equal to the synthesis of a 32-element minimum redundancy virtual array in terms of $L$. In the CDS-based approach, the MIMO arrays are constructed as follows:

$$
\begin{gathered}
\left\{x_{T m}\right\}=D(57,8,1), \\
\left\{x_{R n}\right\}=\left\{b_{n} \cdot 57\right\},
\end{gathered}
$$

where $D(57,8,1)=\{0,1,5,7,17,35,38,49\}$ is a CDS obtained from the Web repositories [24]; $\left\{b_{n}\right\}=\{0,1,4,6\}$ is a 4 -element difference basis for a $[0,6]$ segment (i.e., $P=6$ ). The difference bases $\left\{b_{n}\right\}$ for any $N>3$ always exist with respect to $P$ and can be obtained in $[28,29]$.

Figure 1 shows the behavior of the contiguous virtual array aperture $L$ as a function of the shifted number $\sigma$ by exploiting the CDS-based enumerative shifting procedure. The figure indicates that optimal MR-MIMO layout in terms of $L$ may not be unique. Figures 2(a) and 2(b) show the initial CDS-based MR-MIMO layout $(\sigma=0)$ and the resulting virtual array as well as its coarray, respectively. Figures 2(c) and 2(d) show the optimal MR-MIMO layout $(\sigma=22)$ obtained by the enumerative shifting procedure and the resulting virtual array as well as its coarray, respectively. It is worthwhile to observe that the overall length of the virtual array always exceeds $L$, meaning that several elements of the 

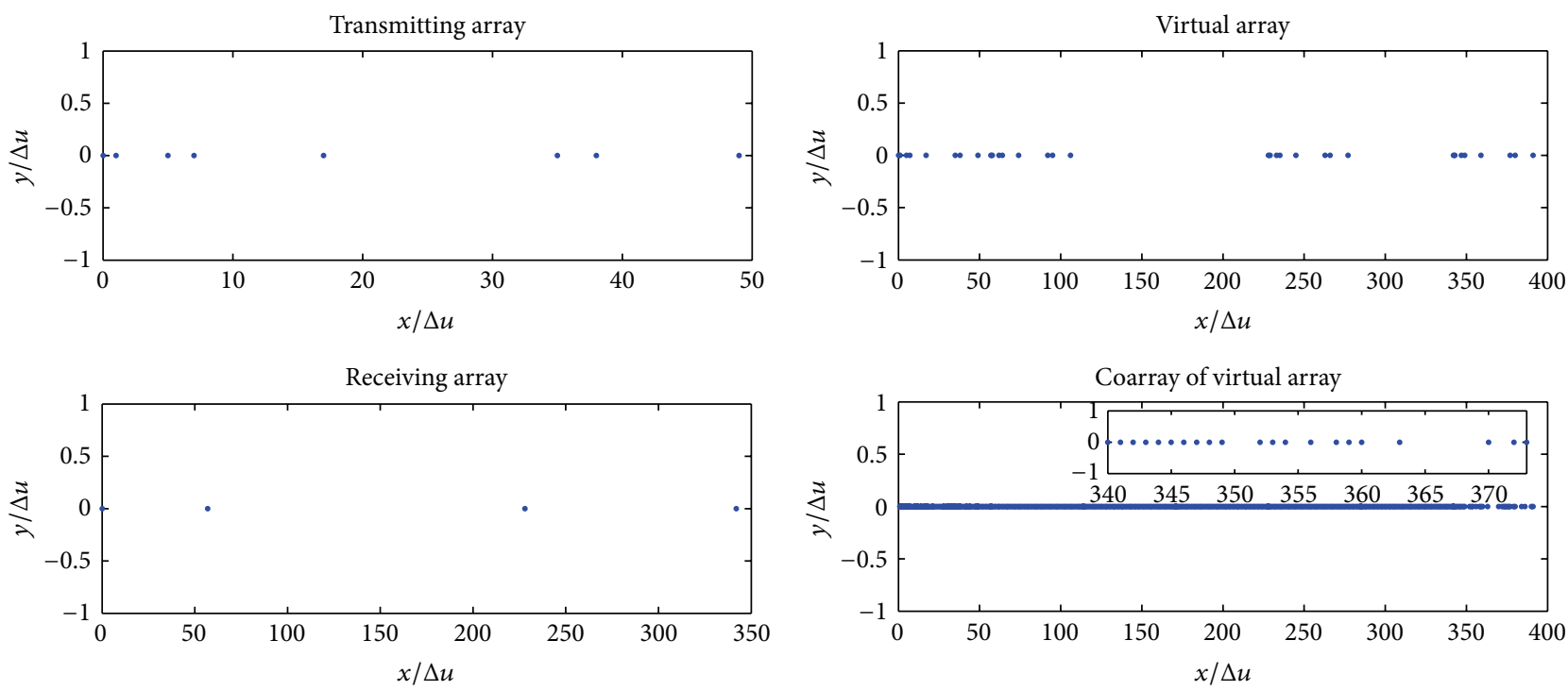

(a)

(b)
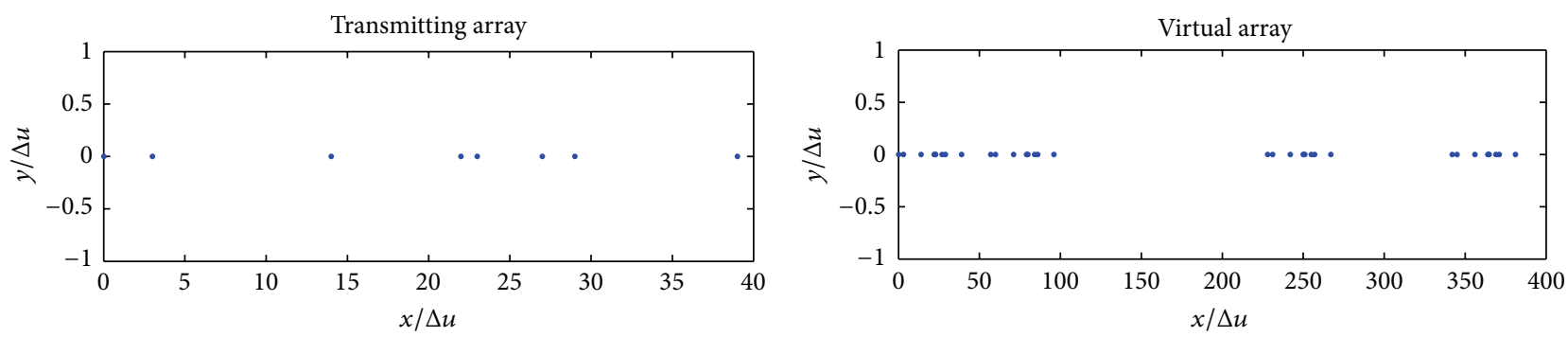

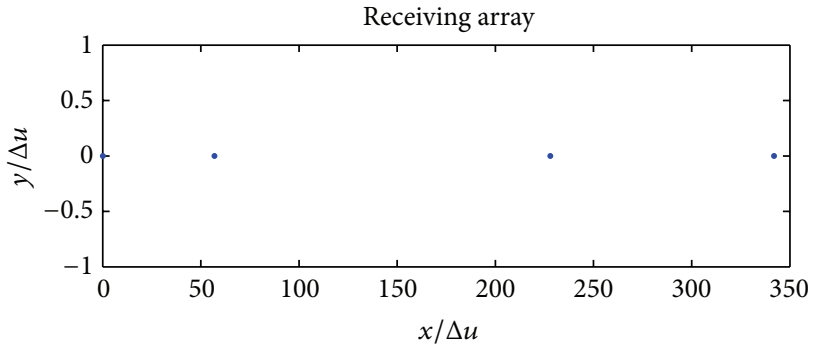

(c)

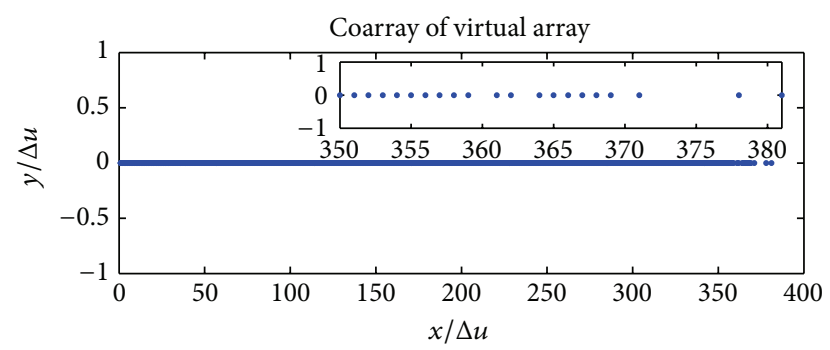

(d)

FIGURE 2: Illustration of an $8 \times 4$ MR-MIMO array synthesis from the initial CDS (the upper row) and the enumerative shifting procedure (the lower row); (a) and (c) the lattice location of the transmitting and receiving arrays; (b) and (d) the synthesized virtual array and its coarray.

virtual array are outside the $[0, L]$ segment. This additional resolution afforded by the excessive overall length might be welcome in some applications, even though the spacing coverage is incomplete above $L$.

Further, in order to show computation efficiency of our CDS-based approach, other MR-MIMO array designs obtained by stochastic optimization methods are also investigated. For MR-MIMO array synthesis problem, the total size of the search space is determined by

$$
N_{t}={ }_{L} C_{M-1} \cdot{ }_{L} C_{N-1},
$$

where ${ }_{p} C_{q}=p ! / q !(p-q) !$ is the number of combinations of $p$ items taken $q$ at a time; $x_{T 1}=0$ and $x_{R 1}=0$ are specified, respectively. Obviously, the size of the search space will exponentially increase with the specified virtual array aperture $L$. Therefore, it is difficult for stochastic optimization methods to find a satisfactory solution (i.e., MR-MIMO array with a large value of $L$ ) after a nonnegligible number of iterations. As an example, Figure 3 shows the convergence rate of the PSO-based search when finding an MR-MIMO solution with $L=294$ (where the size of the search space is of the order of $10^{20}$ according to (23)). Note that, except for the need of a large number of iterations (because of the rigorous terminal condition $H\left(D_{V A}\right)=0$ ), the fitness (i.e., $H\left(D_{V A}\right)$ ) computation for each individual at each iteration is time consuming. However, only less than $M^{2} \times(M-$ 1) $=428$ evaluations are required for identifying an optimal 
TABLE 1: Comparisons of different MR-MIMO layouts with their characteristics $(M=8, N=4)$.

\begin{tabular}{llll}
\hline & PSO-based & Initial CDS-based & Optimal CDS-based \\
& MR-MIMO array & MR-MIMO array & MR-MIMO array \\
\hline Array & $\left\{x_{T m}\right\}=\{0,2,3,8,17,24,28,47\}$ & $\left\{x_{T m}\right\}=\{0,1,5,7,17,35,38,49\}$ & $\left\{x_{T m}\right\}=\{0,3,14,22,23,27,29,39\}$ \\
Locations & $\left\{x_{R n}\right\}=\{0,57,114,285\}$ & $\left\{x_{R n}\right\}=\{0,57,228,342\}$ & $\left\{x_{R n}\right\}=\{0,57,228,342\}$ \\
$L$ & 294 & 349 & 359 \\
$\Delta \theta_{-3 \mathrm{~dB}}(\mathrm{rad})$ & $6.1 \times 10^{-3}$ & $5.1 \times 10^{-3}$ & $5.0 \times 10^{-3}$ \\
$R$ & 1.69 & 1.42 & 1.38 \\
\hline
\end{tabular}

TABLE 2: Comparisons of different MR-MIMO layouts with their characteristics $(M=20, N=10)$.

\begin{tabular}{|c|c|c|}
\hline & $\begin{array}{l}\text { Initial CDS-based } \\
\text { MR-MIMO array }\end{array}$ & $\begin{array}{l}\text { Optimal CDS-based } \\
\text { MR-MIMO array }\end{array}$ \\
\hline Array & $\begin{aligned}\left\{x_{T m}\right\}= & \{0,1,4,52,62,64,84,131,154,162,180,187, \\
& 273,294,300,309,328,339,344,368\}\end{aligned}$ & $\begin{aligned}\left\{x_{T m}\right\}= & \{0,21,27,36,55,66,71,95,108,109,112,160 \\
& 170,172,192,239,262,270,288,295\}\end{aligned}$ \\
\hline Locations & $\begin{aligned}\left\{x_{R n}\right\}= & \{0,381,1143,2286,4953,7620,10287,11811, \\
& 13335,13716\}\end{aligned}$ & $\begin{aligned}\left\{x_{R n}\right\}= & \{0,381,1143,2286,4953,7620,10287,11811 \\
& 13335,13716\}\end{aligned}$ \\
\hline$L$ & 13728 & 13801 \\
\hline$\Delta \theta_{-3 \mathrm{~dB}}(\mathrm{rad})$ & $1.3 \times 10^{-4}$ & $1.29 \times 10^{-4}$ \\
\hline$R$ & 1.45 & 1.44 \\
\hline
\end{tabular}

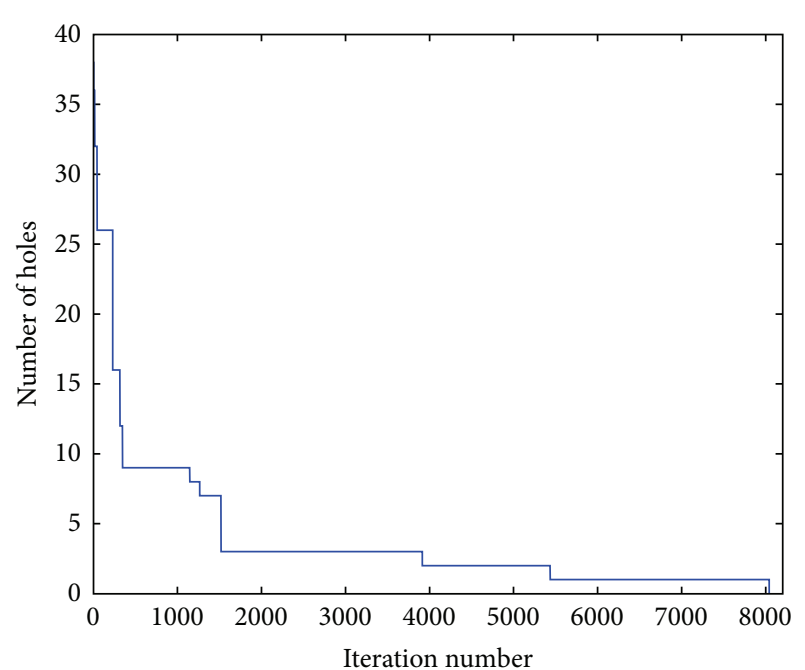

FIGURE 3: Minimum number of holes versus the number of iterations by using the PSO-based search.

$8 \times 4$ MR-MIMO layout in the CDS-based enumerative shifting procedure. Moreover, the fitness computation in this procedure is very simple and requires nearly zero computation time. For summary, different MR-MIMO layouts and their characteristics, such as the maximum contiguous aperture $L$, the angle resolution $\left(\Delta \theta_{-3 \mathrm{~dB}}=0.89 \lambda /(L\right.$. $\Delta u)$ ), and redundancy of the coarray $\left(R={ }_{M N} C_{2} / L=\right.$ $M N(M N-1) / 2 L$ [23]), are listed in Table 1. The comparisons show that by minimizing redundancies in the coarray, the optimized CDS-based MR-MIMO layout has achieved the largest virtual array aperture, meaning that it has the highest spatial resolution and also the largest number of available spatial degrees of freedom [8].

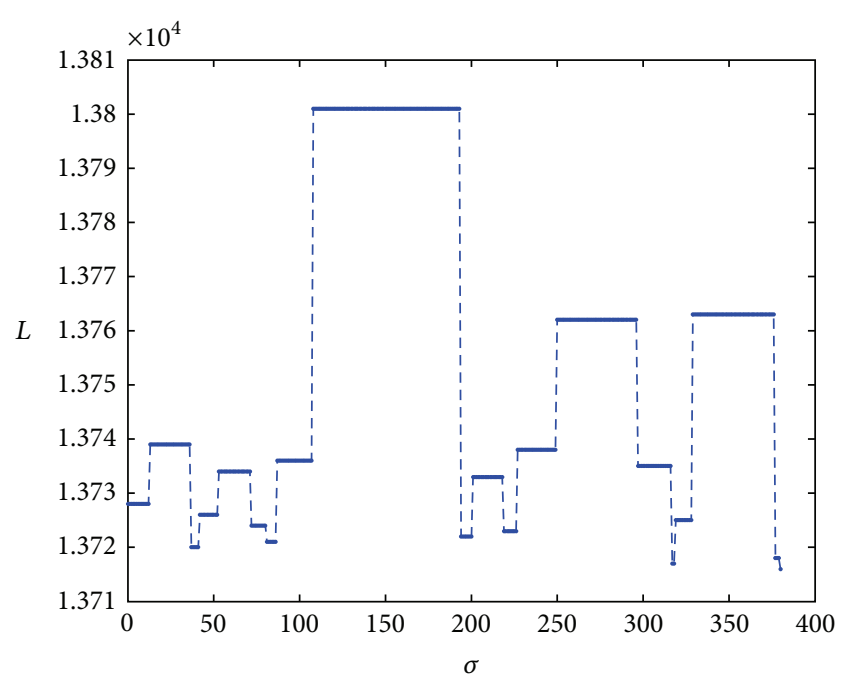

FIGURE 4: CDS-based enumerative shifting procedure: behavior of virtual array aperture $L$ versus evaluated shift $\sigma$ for CDS-based MRMIMO array $(M=20, N=10)$.

The second test case refers to a half wavelength $(\Delta u=$ $0.5 \lambda)$ MIMO radar array with a twenty-element linear transmitting array $(M=20)$ and a ten-element linear receiving array $(N=10)$ in order to validate the large MR-MIMO array synthesis by means of CDSs. The synthesis of a $20 \times 10$ MR-MIMO array is equal to the synthesis of a 200-element minimum redundancy virtual array in terms of $L$. In the CDSbased approach, the MIMO arrays are constructed as follows:

$$
\begin{gathered}
\left\{x_{T m}\right\}=D(381,20,1), \\
\left\{x_{R n}\right\}=\left\{b_{n} \cdot 381\right\},
\end{gathered}
$$



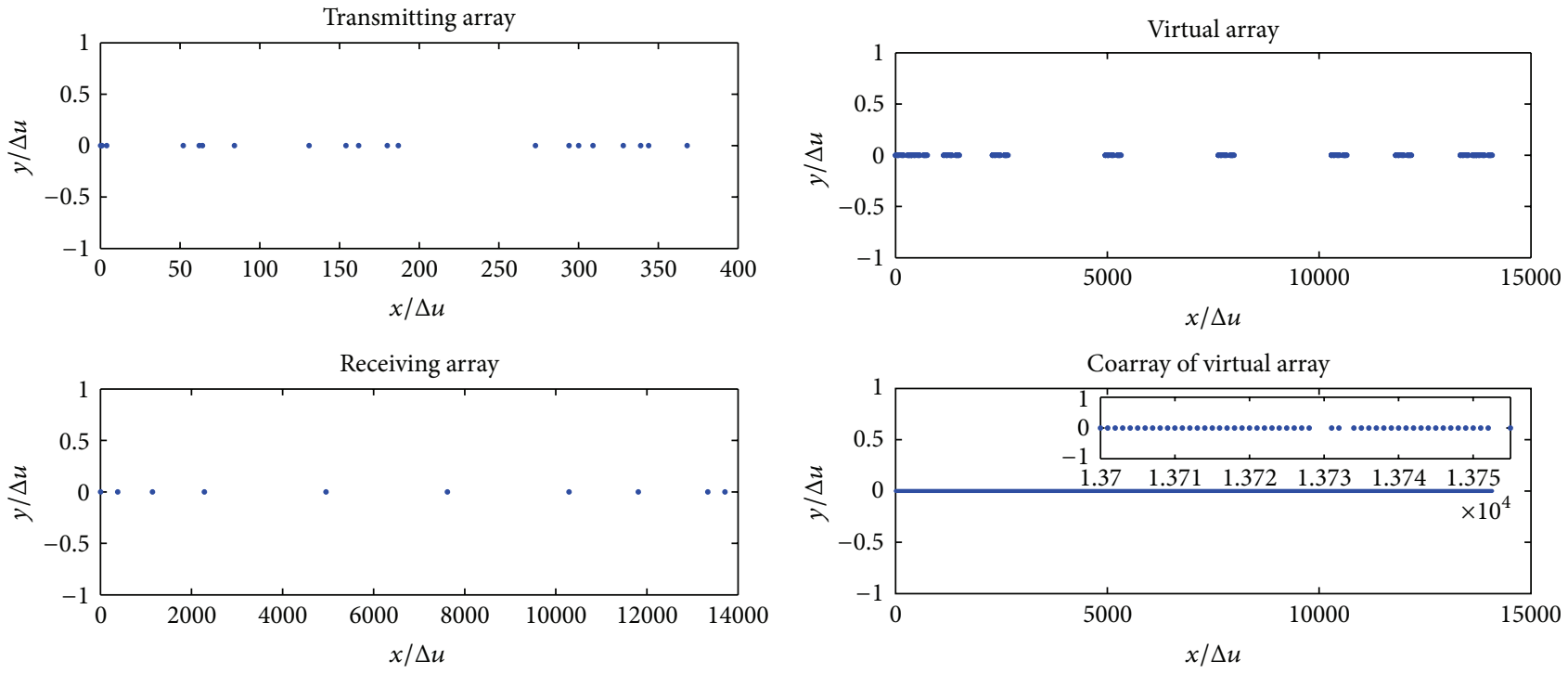

(a)

(b)
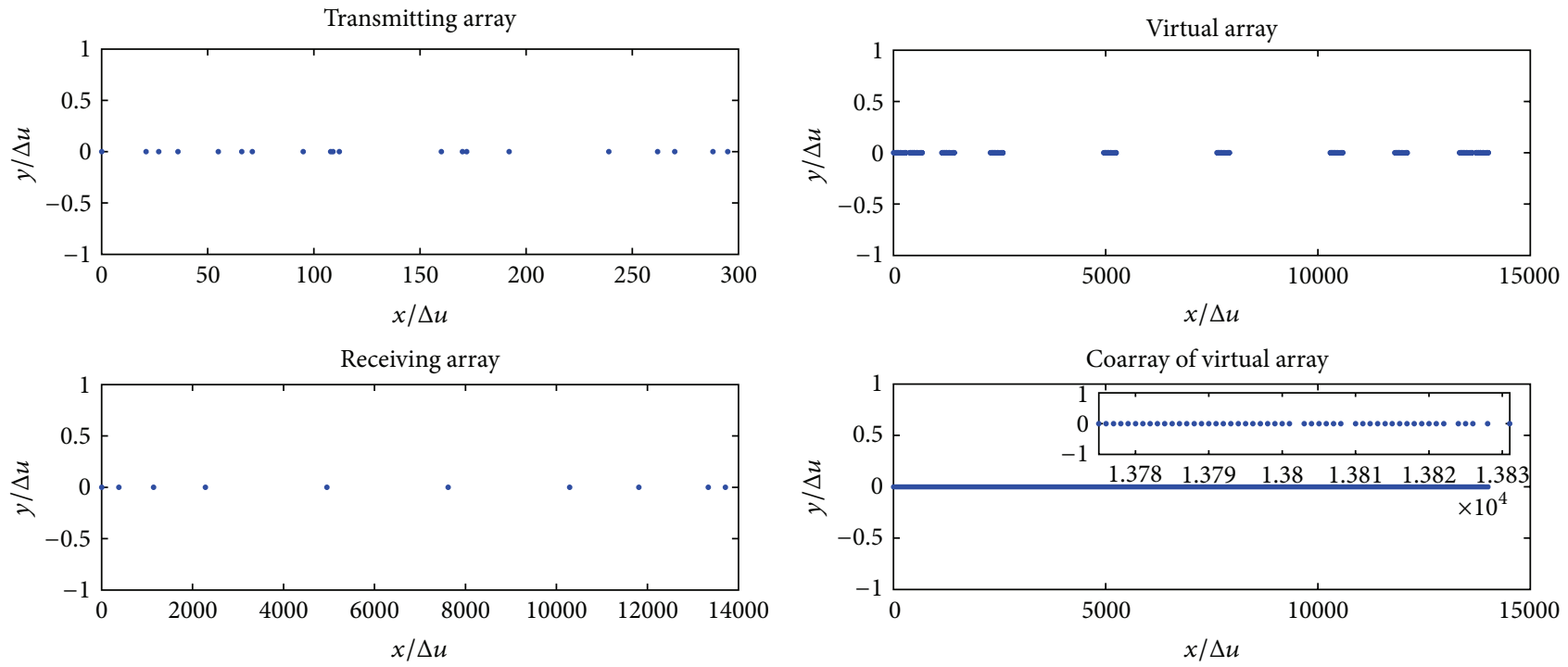

(c)

(d)

FIGURE 5: Illustration of a $20 \times 10$ MR-MIMO array synthesis from the initial CDS (the upper row) and the enumerative shifting procedure (the lower row) (a) and (c) the lattice location of the transmitting and receiving array (b) and (d) the synthesized virtual array and its coarray.

where $D(381,20,1)=\{0,1,4,52,62,64,84,131,154,162$, $180,187,273,294,300,309,328,339,344,368\}$ is a CDS obtained from the Web repositories $[24] ;\left\{b_{n}\right\}=\{0,1,3,6$, $13,20,27,31,35,36\}$ is a 10 -element difference basis for a $[0,36]$ segment (i.e., $P=36$ ). Figure 4 shows the behavior of the contiguous virtual array aperture $L$ as a function of the shifted number $\sigma$ by exploiting the CDS-based enumerative shifting procedure. Figures 5(a) and 5(b) show the initial CDS-based MR-MIMO layout $(\sigma=0)$ and the resulting virtual array as well as its coarray. Figures 5(c) and $5(\mathrm{~d})$ show the optimal MR-MIMO layout $(\sigma=108)$ obtained by the enumerative shifting procedure and the resulting virtual array as well as its coarray, respectively. More specifically, both CDS-based MR-MIMO layouts and their characteristics, such as the maximum contiguous aperture $L$, the angle resolution $\Delta \theta_{-3 \mathrm{~dB}}$, and redundancy of the coarray $R$, are listed in Table 2 . Note that, in this test case, an exhaustive search or a stochastic optimizer barely seems powerless for finding MR-MIMO arrays within a reasonable time due to the extremely huge search space (e.g., given $L=10000$, the size of the search space will be of the order $10^{89}$ according to (23)) and the rigorous constraint function. However, only less than 8000 evaluations are required for identifying an optimal $20 \times 10$ MR-MIMO layout in the CDS-based enumerative shifting procedure. Moreover, the fitness computation in this procedure takes nearly zero computation time. In conclusion, the CDS-based enumerative shifting procedure is computationally efficient, 
and its advantage in optimization efficiency will become more significant with the increased scale of MR-MIMO array synthesis.

\section{Conclusion and Discussion}

As a kind of deterministic thinning methodologies, the CDSbased approach presents several potentials over stochastic optimizers (e.g., GAs and PSO) or trial-and-test random techniques such as the computational efficiency and the predictable array performances (e.g., virtual array aperture) thanks to the analytical features of the arising CDS-based MIMO arrays. To identify the optimal MR-MIMO layout, a numerically efficient procedure has been developed in this paper, which is limited to an enumerative shifting of reference sequences available in Web repositories. Selected examples have been analyzed to point out the computational effectiveness of the CDS-based MR-MIMO array synthesis.

Further studies could be expected from the following aspects.

(1) Other analytical sequences (e.g., ADSs, Hadamard Difference Sets [30], and McFarland difference sets [31]) with attractive features (e.g., low-level autocorrelation and cyclic shifting) could be investigated to verify their advantages and potentialities for MIMO array designs. Indeed, it has been recently shown that likewise CDS placements, but in a theoretically wider admissible set of configurations, ADSs can be profitably exploited to maximize channel capacity in MIMO communication applications [32].

(2) The integration of analytical sequences and global optimizers for improved exploration efficiency is still a work in progress.

\section{Acknowledgments}

This work was supported in part by the National Science Foundation of China under Grant no. NSFC61201086, the Doctoral Fund of the Ministry of Education of China under Grant no. 20110162120044, and the China Postdoctoral Science Foundation under Grant no. 2012T50660.

\section{References}

[1] K. W. Forsythe, D. W. Bliss, and G. S. Fawcett, "Multipleinput multiple-output (MIMO) radar: performance issues," in Proceedings of the 38th Asilomar Conference on Signals, Systems and Computers, pp. 310-315, November 2004.

[2] E. Fishler, A. Haimovich, R. Blum, L. Cimini, D. Chizhik, and R. Valenzuela, "Performance of MIMO radar systems: advantages of angular diversity," in Proceedings of the 38th Asilomar Conference on Signals, Systems and Computers, pp. 305-309, November 2004.

[3] E. Fishler, A. Haimovich, R. S. Blum, L. J. Cimini Jr., D. Chizhik, and R. A. Valenzuela, "Spatial diversity in radars-models and detection performance," IEEE Transactions on Signal Processing, vol. 54, no. 3, pp. 823-838, 2006.

[4] D. W. Bliss and K. W. Forsythe, "Multiple-input multipleoutput (MIMO) radar and imaging: degrees of freedom and resolution," in Proceedings of the 37th Asilomar Conference on Signals, Systems and Computers, pp. 54-59, November 2003.

[5] C.-Y. Chen and P. P. Vaidyanathan, "MIMO radar space-time adaptive processing using prolate spheroidal wave functions," IEEE Transactions on Signal Processing, vol. 56, no. 2, pp. 623635, 2008.

[6] Y. Qu, G. S. Liao, S. Q. Zhu, X. Y. Liu, and H. Jiang, "Performance analysis of beamforming for MIMO radar," Progress in Electromagnetics Research, vol. 84, no. 2, pp. 123-134, 2008.

[7] W.-Q. Wang, "Virtual antenna array analysis for MIMO synthetic aperture radars," International Journal of Antennas and Propagation, vol. 2012, Article ID 587276, 10 pages, 2012.

[8] W.-Q. Wang, H. Shao, and J. Cai, "MIMO antenna array design with polynomial factorization," International Journal of Antennas and Propagation, vol. 2013, 9 pages, 2013.

[9] M. Karaman, I. O. Wygant, Ö. Oralkan, and B. T. Khuri-Yakub, "Minimally redundant 2-D array designs for 3-D medical ultrasound imaging," IEEE Transactions on Medical Imaging, vol. 28, no. 7, pp. 1051-1061, 2009.

[10] P. Pal and P. P. Vaidyanathan, "Nested arrays: a novel approach to array processing with enhanced degrees of freedom," IEEE Transactions on Signal Processing, vol. 58, no. 8, pp. 4167-4181, 2010.

[11] C.-Y. Chen and P. P. Vaidyanathan, "Minimum redundancy MIMO radars," in Proceedings of the IEEE International Symposium on Circuits and Systems (ISCAS '08), pp. 45-48, May 2008.

[12] A. T. Moffet, "Minimum-redundancy linear arrays," IEEE Transactions on Antennas and Propagation, vol. 16, pp. 172-175, 1968.

[13] S. U. Pillai, Y. Bar-Ness, and F. Haber, "A new approach to array geometry for improved spatial spectrum estimation," Proceedings of the IEEE, vol. 73, no. 10, pp. 1522-1524, 1985.

[14] K. Rezer, W. Gropengießer, and A. F. Jacob, "Particle swarm optimization of minimum-redundancy MIMO arrays," in Proceedings of the German Microwave Conference (GeMiC '11), March 2011.

[15] D. G. Leeper, "Isophoric arrays-massively thinned phased arrays with well-controlled sidelobes," IEEE Transactions on Antennas and Propagation, vol. 47, no. 12, pp. 1825-1835, 1999.

[16] S. Caorsi, A. Lommi, A. Massa, and M. Pastorino, "Peak sidelobe level reduction with a hybrid approach based on GAs and difference sets," IEEE Transactions on Antennas and Propagation, vol. 52, no. 4, pp. 1116-1121, 2004.

[17] G. Oliveri, M. Donelli, and A. Massa, "Linear array thinning exploiting almost difference sets," IEEE Transactions on Antennas and Propagation, vol. 57, no. 12, pp. 3800-3812, 2009.

[18] G. Oliveri, L. Manica, and A. Massa, "ADS-based guidelines for thinned planar arrays," IEEE Transactions on Antennas and Propagation, vol. 58, no. 6, pp. 1935-1948, 2010.

[19] G. Oliveri and A. Massa, "Fully interleaved linear arrays with predictable sidelobes based on almost difference sets," IET Radar, Sonar and Navigation, vol. 4, no. 5, pp. 649-661, 2010.

[20] G. Oliveri and A. Massa, "Genetic algorithm (GA)-enhanced almost difference set (ADS)-based approach for array thinning," IET Microwaves, Antennas and Propagation, vol. 5, no. 3, pp. 305-315, 2011.

[21] G. Oliveri, F. Caramanica, and A. Massa, "Hybrid ADS-based techniques for radio astronomy array design," IEEE Transactions on Antennas and Propagation, vol. 59, no. 6, pp. 1817-1827, 2011. 
[22] M. Carlin, G. Oliveri, and A. Massa, "On the robustness to element failures of linear ADS-thinned arrays," IEEE Transactions on Antennas and Propagation, vol. 59, no. 12, pp. 4849-4853, 2011.

[23] J. Dong, Q. Li, and W. Guo, "A combinatorial method for antenna array design in minimum redundancy MIMO radars," IEEE Antennas and Wireless Propagation Letters, vol. 8, pp. 11501153, 2009.

[24] La Jolla Cyclic Difference Set Repository, http://www.ccrwest .org/diffsets.html.

[25] L. D. Baumert, Cyclic Difference Sets, Springer, New York, NY, USA, 1971.

[26] M. Hall Jr, Combinatorial Theory, Wiley, New York, NY, USA, 2nd edition, 1986.

[27] J. Leech, "On the representation of $1,2, \ldots, n$ by differences," Journal London Mathematical Society, vol. 31, pp. 160-169, 1956.

[28] D. A. Linebarger, I. H. Sudborough, and I. G. Tollis, "Difference bases and sparse sensor arrays," IEEE Transactions on Information Theory, vol. 39, no. 2, pp. 716-721, 1993.

[29] J. Dong, Q. Li, R. Jin, Y. Zhu, Q. Huang, and L. Gui, "A method for seeking low-redundancy large linear arrays in aperture synthesis microwave radiometers," IEEE Transactions on Antennas and Propagation, vol. 58, no. 6, pp. 1913-1921, 2010.

[30] L. E. Kopilovich, "Square array antennas based on Hadamard difference sets," IEEE Transactions on Antennas and Propagation, vol. 56, no. 1, pp. 263-266, 2008.

[31] G. Oliveri, F. Caramanica, C. Fontanari, and A. Massa, "Rectangular thinned arrays based on McFarland difference sets," IEEE Transactions on Antennas and Propagation, vol. 59, no. 5, pp. 1546-1552, 2011.

[32] G. Oliveri, F. Caramanica, M. D. Migliore, and A. Massa, "Synthesis of non-uniform MIMO arrays through combinatorial sets," IEEE Antennas and Wireless Propagation Letters, vol. 11, pp. 728-731, 2012. 

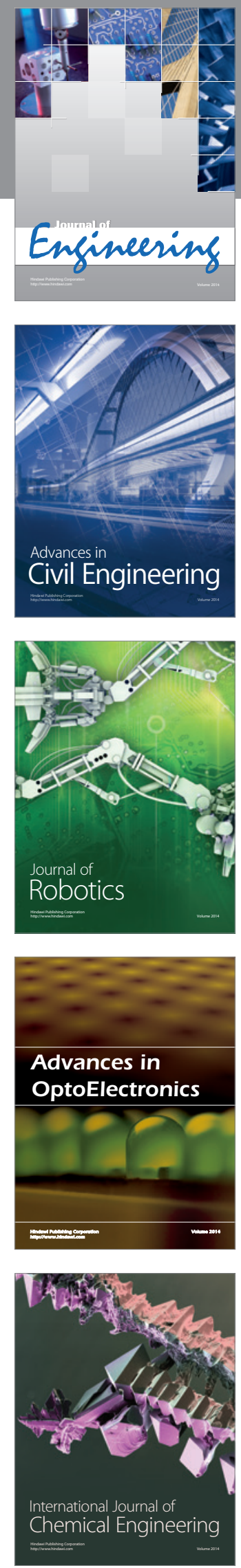

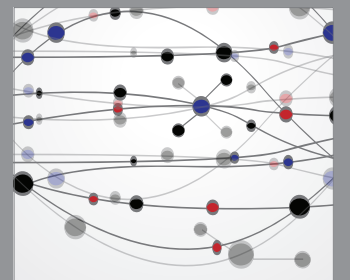

The Scientific World Journal
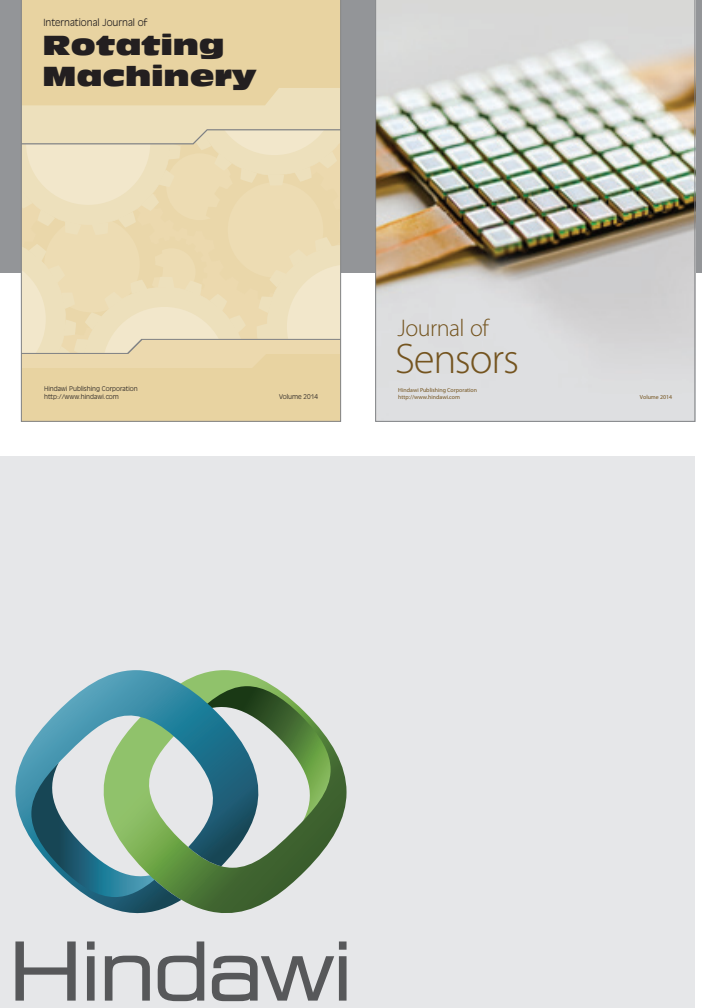

Submit your manuscripts at http://www.hindawi.com
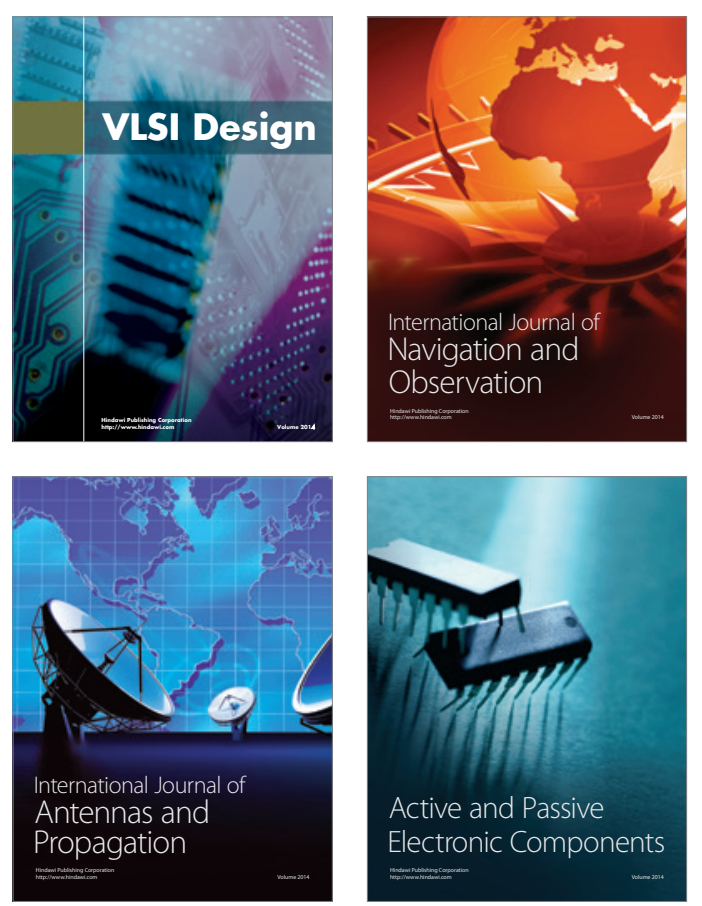
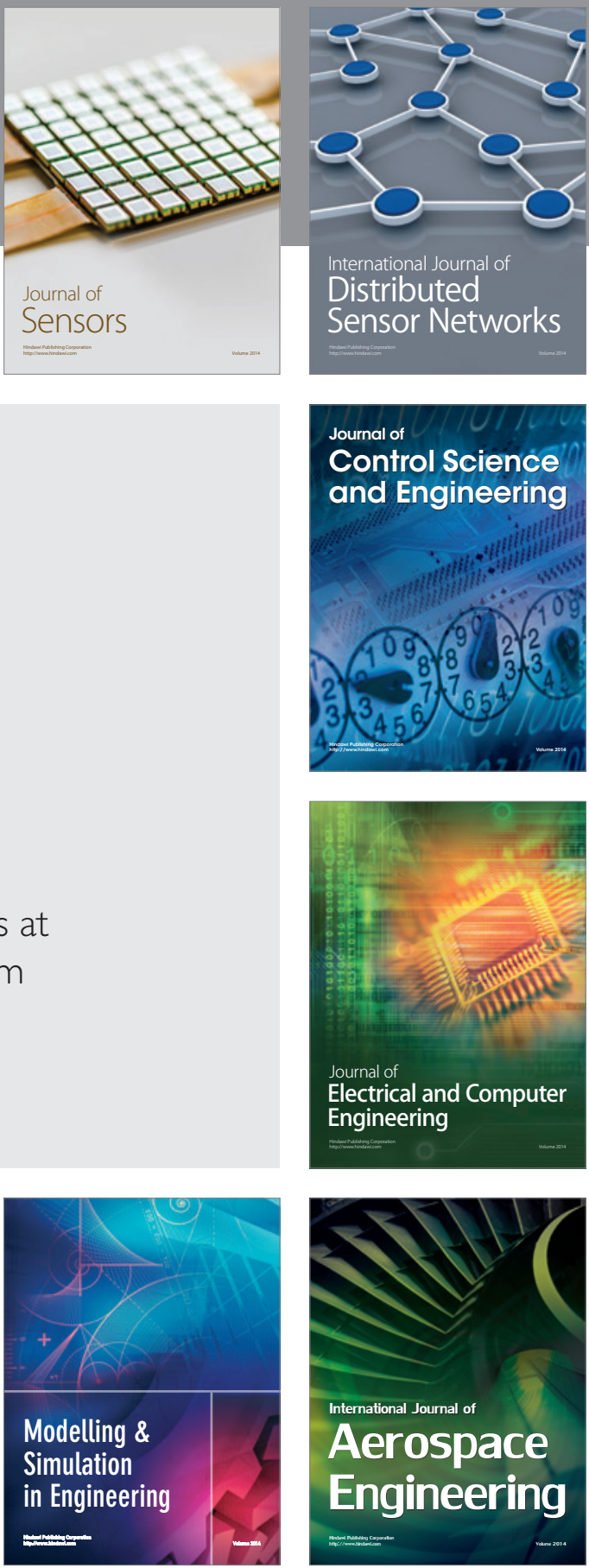

Journal of

Control Science

and Engineering
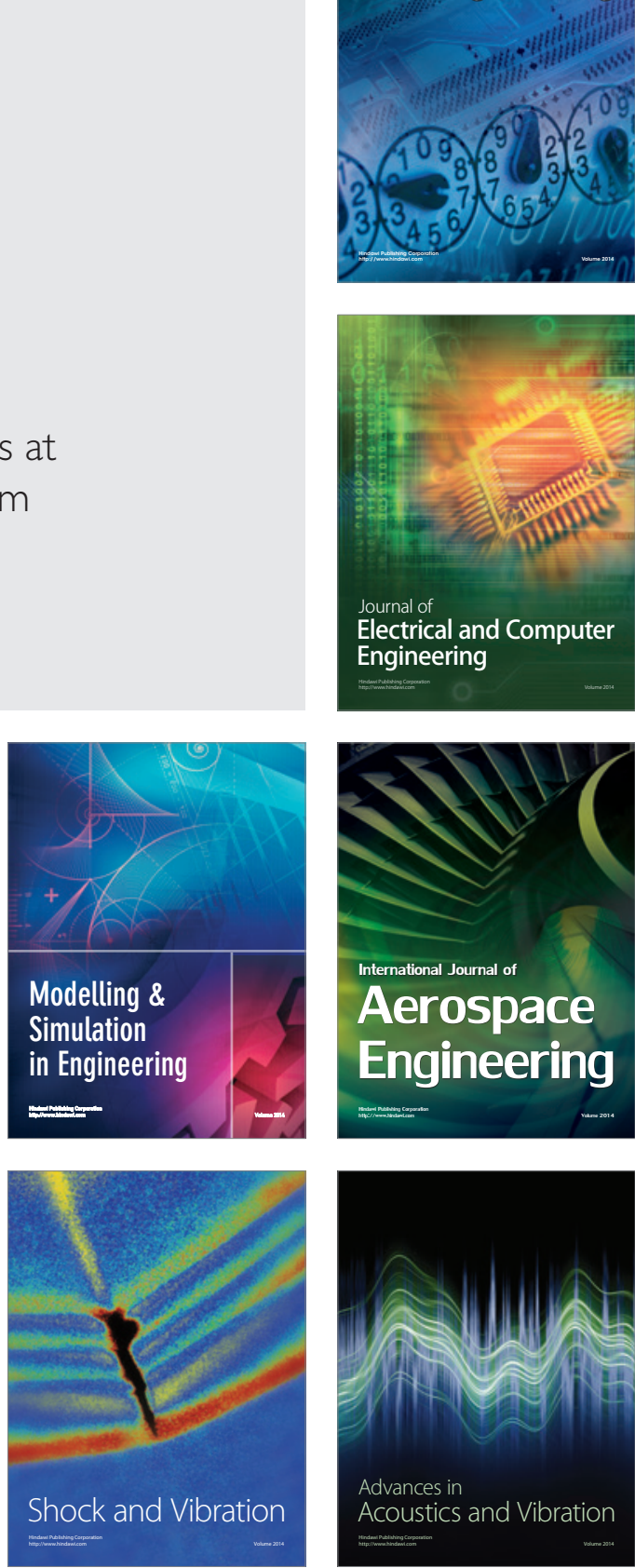